\title{
Compreensão da leitura textual e sua relação com as funções executivas
}

\section{Text reading comprehension and its relationship with executive functions}

\author{
Jerusa Fumagalli de Salles ${ }^{1}$ \\ Fraulein Vidigal de Paula ${ }^{2}$
}

\begin{abstract}
RESUMO
A leitura é uma atividade complexa que envolve diversos processos, tais como identificação de letras, reconhecimento de palavras, acesso ao significado, integração sintática e semântica. A identificação/reconhecimento de palavras apesar de necessária para compreender um texto não é suficiente. Neste artigo o foco será a relação entre compreensão textual e as funções executivas. São abordados brevemente alguns dos principais modelos e alguns instrumentos de avaliação de compreensão de leitura textual. Em seguida, é apresentado o conceito, componentes e principais instrumentos de avaliação das funções executivas. Por último, apresentamos uma síntese reflexiva sobre estudos recentes que investigam as relações entre funções executivas e compreensão de texto. Tanto em estudos nacionais, quanto internacionais estas relações têm sido evidenciadas, assim como os efeitos positivos de programas para a promoção do desenvolvimento das funções executivas, desde a educação infantil, as quais podem contribuir posteriormente também para auxiliar na compreensão de textos.
\end{abstract}

Palavras-chave: compreensão de texto; leitura; funções executivas; memória operacional; linguagem.

DOI: $10.1590 / 0104-4060.48332$

1 Universidade Federal do Rio Grande do Sul. Instituto de Psicologia. Porto Alegre, Rio Grande do Sul, Brasil. Rua Ramiro Barcelos, n² 2.600. CEP: 90035-003.E-mail: jerusafs@yahoo. com.br

2 Universidade de São Paulo. Instituto de Psicologia. Programa de Pós-Graduação em Psicologia Escolar e do Desenvolvimento Humano. São Paulo, São Paulo, Brasil. Av. Prof. Mello Moraes, nº 1.721. CEP: 05508-030.E-mail: fraulein@usp.br 


\begin{abstract}
Reading is a complex task that involves diverse processes such as the identification of letters, recognition of words, access to meanings, and syntactic and semantic integration. Even though the identification/ recognition of words is necessary to understand a text, it is not sufficient. This paper focuses on the relationship between textual understanding and executive functions. Some of the main models and instruments used to assess textual reading understanding are briefly approached. Afterwards, the concept, components and main instruments to assess executive functions are presented. Finally, a reflective synthesis of recent studies investigating the relationship between executive functions and textual understanding is presented. These relationships have been reported in Brazilian and international studies as well as the positive effects of programs promoting the development of executive functions beginning in child education, which also later can contribute to support reading comprehension.
\end{abstract}

Keywords: reading comprehension; reading; executive functions; working memory; language.

A leitura é uma atividade complexa que envolve diversos processos: identificação de letras, reconhecimento de palavras, acesso ao significado, integração sintática e semântica. A identificação/reconhecimento de palavras apesar de necessária para compreender um texto não é suficiente. Quanto mais rápida é a identificação de cada palavra, a memória de trabalho tem mais disponibilidade de recursos para realizar operações de análise sintática, de integração semântica dos constituintes da frase e de integração das frases na organização textual, processos importantes para a compreensão da leitura. (MORAIS, 1996). A compreensão leitora tem forte relação também com exposição à escrita, conhecimento do vocabulário, metacognição, habilidades semânticas (NATION; SNOWLING, 1998), consciência sintática (GAUX; GOMBERT, 1999), além de processos que também são usados para a compreensão da linguagem oral, incluindo o conhecimento geral sobre o tema tratado no texto. Neste artigo o foco recai sobre a relação entre compreensão leitora e funções executivas. São abordados brevemente alguns dos principais modelos de compreensão leitora e alguns instrumentos de avaliação de compreensão de leitura textual. Em seguida, é apresentado o conceito, componentes e principais instrumentos de avaliação das funções executivas. Serão discutidos os diferentes subprocessos de funções executivas potencialmente envolvidos na compreensão. Por último, será feita uma síntese reflexiva sobre estudos recentes que investigam as relações entre funções executivas e compreensão de texto. 


\section{Alguns modelos de compreensão em leitura textual e exemplos de instrumentos de avaliação}

Corso, Sperb e Salles (2013a) apresentam uma revisão de alguns modelos de processamento da compreensão de leitura textual. Dentre estes citam o modelo de Kintsch $(1988,1998)$, que preconiza que na leitura há a construção de uma versão literal do texto - o texto-base, que se organiza em dois níveis estruturais: a microestrutura (rede de proposições, que são unidades de significados, formada a partir da combinação dos significados das palavras) e a macroestrutura, formada a partir do reconhecimento dos tópicos globais do texto e suas inter-relações. (KINTSCH; RAWSON, 2005). A partir dessa organização ocorre a integração de informação do texto com o conhecimento prévio relevante, permitindo a construção de um modelo mental da situação descrita pelo texto - o modelo da situação. As inferências ligam informações do próprio texto entre si, ou relacionam informação do texto com o conhecimento prévio. Estratégias de resolução de problemas (resumo de informação relevante, ativação do esquema do tipo de texto que está sendo lido) também entram em jogo na fase de integração. A compreensão exige a extrapolação do texto-base em direção ao modelo mental, a partir da conexão da informação do texto com as estruturas de conhecimento existentes. (KINTSCH; RAWSON, 2005). Os vários processamentos envolvidos na compreensão demandam memória de trabalho. $\mathrm{O}$ executivo central da memória de trabalho parece diretamente relacionado com a compreensão. (CARRETTI et al., 2009).

Outro modelo que pode ser complementar a esse é o de Trabasso (TRABASSO; VAN DEN BROEK; SUH, 1989), que propôs um modelo específico para textos narrativos, segundo o qual as inferências causais conectam as unidades do texto definidas como cláusulas (que correspondem aos eventos da narrativa). Tais inferências operam através de trechos distantes do texto, conectando-os em forma de rede. Assim, o texto narrativo é representado como uma rede causal de cláusulas categorizadas e das relações entre elas. Na estrutura causal em forma de rede, os nodos correspondem às cláusulas, enquanto as conexões entre os nodos são as relações causais entre os eventos.

Dentre os instrumentos de avaliação da compreensão leitora, há aqueles que usam a técnica do Cloze, os que se baseiam na evocação livre do texto lido, os que usam resposta a questões (abertas ou fechadas) sobre o texto e os que combinam mais de uma forma em uma mesma tarefa. A técnica de Cloze, criada por Taylor (1953), identifica a habilidade do leitor de integrar a informação impressa com o conhecimento que tem da estrutura da língua. (CHANCE, 
1985). Por exemplo, o teste proposto por Santos (2009) consiste em dois textos compostos de 204 palavras, cada, mas níveis de dificuldade diferentes. A técnica de Cloze consiste na omissão de cada quinta palavra do texto. O examinando deve preencher com uma palavra que considere mais adequada ao contexto.

O Teste de Leitura de Compreensão de Sentenças (TELCS) (VILHENA et al., 2016) avalia a compreensão leitora por meio da fluência da leitura silenciosa de sentenças em crianças do $2^{\circ}$ ao $5^{\circ}$ ano do ensino fundamental e pode ser aplicado coletivamente. A criança é instruída a ler sentenças que não possuem a última palavra e deve escolher a palavra que melhor completa a frase. O teste possui normas por idade e ano escolar para as crianças brasileiras. A tarefa de compreensão de leitura textual proposta em Corso et al. (2015) é composta por dois textos narrativos para a avaliação de crianças de $1^{\circ}$ ao $6^{\circ}$ ano do ensino fundamental. A história "A Coisa" possui aproximadamente 200 palavras, com extensão e níveis de legibilidade apropriados para a escolaridade da amostra (até três anos completos de estudo) e "O Coelho e o Cachorro" com 341 palavras, para ser utilizado entre o $4^{\circ}$ e $6^{\circ}$ ano escolares. (CORSO; SPERB; SALLES; 2012; SALLES; PARENTE, 2004). A criança é instruída a ler a história atentamente e são informados os objetivos desta leitura, ou seja, que ela será posteriormente solicitada a recontar (evocar livremente) a história e, em seguida, a responder a questões sobre a história. $\mathrm{O}$ questionário referente à história lida é composto por dez questões de múltipla escolha, com quatro opções de resposta. Metade das perguntas relaciona-se com memória para eventos e personagens descritos na própria história, e a outra metade avalia compreensão inferencial. $\mathrm{O}$ examinador lê as questões e as respectivas opções de resposta, enquanto a criança acompanha a leitura visualmente. Por fim, o participante indica a resposta que considera correta. As normas para a população brasileira são expostas conforme anos completos de estudo e tipo de escola. (CORSO et al., 2015).

Há também a avaliação da compreensão leitora de textos expositivos (SARAIVA; MOOJEN; MUNARSKI, 2005), composta por vários textos, com diferentes formas de organização global. É possível calcular a fluência de leitura, verificando-se a média de palavras lidas por minuto. Após a leitura, o examinando relata o que leu e reponde a algumas questões.

\section{Funções executivas, seus componentes e instrumentos de avaliação}

As funções executivas dizem respeito a processos e habilidades cognitivas que compõem a competência das pessoas de exercer com sucesso controle vo- 
luntário, independente e orientado por metas sobre seu funcionamento, estando menos sujeitas às contingências ambientais. Desse modo, são aquelas que permitem a uma pessoa orientar e gerenciar suas funções cognitivas, emocionais e comportamentais de modo mais consciente, flexível e adaptativo frente às demandas internas e externas. Essas funções são ativadas em condições em que o processamento automático não é suficiente ou adequado, em situações de formação de julgamentos, tomada de decisões, solução de problemas complexos, e em atividades como estudar, aprender algo novo, resolver conflitos interpessoais. Pessoas com essas funções comprometidas teriam dificuldades de adotar estratégias de planejamento, organização, condução e monitoramento de suas atividades. (LEZAK; HOWIESON; LORING, 2004; MENEZES; GODOY et al., 2012; CORSO et al., 2013; SEABRA et al., 2014b).

Em um estudo de revisão, Corso et al. (2013) identificaram um paralelo ao conceito de metacognição, em uma abordagem cognitiva, para designar a capacidade de autogerenciamento dos recursos cognitivos. O termo "funções executivas" teria sido primeiramente apresentado no manual de avaliação neuropsicológica de Muriel Deutsch Lezak, Howieson e Loring (2004), ao qual se sucederam várias publicações com diferentes definições e modelos para representar as funções executivas. Essas têm sido estudadas em relação a diferentes faixas etárias (CARREIRO et al., 2014), bem como em relação a diferentes domínios, tais como raciocínio matemático, teoria da mente e leitura. (CANTIN et al., 2016). Em um estudo brasileiro com pré-escolares, Pazeto, Seabra e Dias (2014) evidenciaram que as funções executivas se expandem de um ano ao outro já nesta etapa de vida e se relacionam com avanços também na linguagem oral.

Vários modelos teóricos e experimentais têm sido propostos e testados a respeito de quais seriam e como se relacionariam as habilidades que compõem as funções executivas. Entre estes componentes estariam a volição, a atenção seletiva, a resistência à distração ou controle inibitório, a flexibilidade cognitiva, afetiva e comportamental, além de memória operacional, metacognição e autorregulação, a capacidade de eleger objetivos, planejar-se, realizar automonitoramento, autoavaliação e comporta-se orientado por metas e propósitos. (SEABRA et al., 2014b; DIAMOND, 2013; CORSO et al., 2013). Seabra et al. (2014b) avaliam que esta diversidade de processos associados às funções executivas deve-se a diferenças metodológicas entre os estudos, referentes à faixa etária, tipos e quantidade de instrumentos de avaliação, embora tenham em comum a compreensão de que estas não são formadas por um único fator.

As funções executivas talvez possam ser consideradas como o agrupamento mais amplo desta diversidade de processos que exigem diferentes níveis de consciência (LOGAN, 1988), deliberação e regulação. Tomadas, assim, em um contínuo, pode-se supor que alguns dos aspectos das funções executivas surgem 
mais cedo, de modo menos elaborado. Mais tarde, se tornariam mais elaboradas, especializadas e diversificadas no processo de desenvolvimento. Parte destas habilidades, as mais elaboradas e coordenadas, exigiriam mais experiência, aprendizagens e desenvolvimento para se manifestarem. (DIAMOND, 2013). No entanto, é mais recorrente na literatura a consideração de três processos cognitivos como componentes principais das funções executivas, sendo eles a inibição, a memória operacional e a flexibilidade, o que tem sido corroborado por estudos recentes, com uso de análise fatorial e equações estruturais. (MYAKE et al., 2000; CANTIN et al., 2016; CORSO et al., 2016).

O primeiro desses componentes, a inibição, envolve atenção seletiva e controle inibitório frente a estímulos externos não pertinentes à atividade principal em curso, bem como à estimulação interna, como linhas de pensamento concorrentes à meta principal. A atenção seletiva pode ser definida como a capacidade de selecionar um estímulo e manter-se concentrada a este, enquanto se ignora outros presentes no mesmo contexto. (STERNBERG, 2010). O controle inibitório envolve autocontrole na resistência a distrações, cansaço ou outras motivações, para manter-se naquilo que precisa ser conduzido e concluído. Envolve também resistir a uma primeira percepção de um problema para chegar a uma interpretação e solução mais apuradas, escapando de lapsos de atenção associados a processos automáticos. O domínio pouco desenvolvido desta habilidade resultaria em impulsividade, intolerância à espera, logo, em um comportamento mais orientado pelas contingências ambientais e menos autodirigido e autorregulado. (Seabra et al., 2014a).

Outro componente seria a memória operacional ou de trabalho, que mantém representações e recursos cognitivos ativos, pertinentes a uma atividade em curso, retidos durante certo tempo necessário à sua realização. Coordena informações derivadas de processos ascendentes, registrados da situação presente, bem como ascendentes, pela recuperação de conhecimento relevante da memória de longo prazo. (BADDELEY, 2000). É considerada uma função executiva na medida em que permite que este conteúdo seja processado em relação a um propósito, a retenção de informações novas, a coordenação de diferentes informações sequenciais, de ideias, além da produção de inferências, ao permitir a coordenação de conhecimentos prévios e aqueles disponíveis na situação. Também tem a função de facilitar a atenção sustentada, o monitoramento da atividade em curso frente a seus objetivos e a flexibilidade no uso destes elementos durante o desempenho da tarefa.

Por último, a flexibilidade na alternância da atenção a diferentes informações e perspectivas seria um outro aspecto das funções executivas. A flexibilidade também pode ocorrer na mudança de estratégias adotadas em uma atividade que está sendo realizada, como um ajustamento para sucesso em sua conclu- 
são. De um modo geral, envolve mudanças e alternâncias no processamento cognitivo em curso. (SEABRA et al., 2014b, DIAMOND, 2013). Desta forma, relaciona-se à ativação e desativação de representações na memória operacional. $\mathrm{Na}$ compreensão oral ou textual, afetaria a capacidade de coordenar diferentes sequências de informação e pontos de vista, levantar hipóteses, considerar mais de um significado possível para uma palavra ou expressão.

As funções executivas seriam necessárias a uma série de tarefas cotidianas que exigem desenvolvimento da autonomia, intencionalidade, persistência e autorregulação, tais como as requeridas na leitura proficiente, realizada com fluência e compreensão. Seriam também influenciadas pelas demandas criadas por estas mesmas tarefas. Estas funções podem ser promovidas em um contexto de instrução. (DIAS; SEABRA, 2013).

Em termos de avaliação, Carreiro et al. (2014) apresentaram um panorama dos testes e baterias adaptados ou desenvolvidos para avaliação de funções executivas no Brasil. Foram identificados um total de 15 instrumentos utilizados em mais de um estudo, sendo quatro validados e disponíveis para uso com parâmetros normativos. Estes testes em geral são aplicáveis a várias faixas etárias, podem avaliar funções executivas simples ou independentes (memória operacional fonológica, atenção seletiva e flexibilidade) ou funções executivas complexas, ou seja, quando o teste mobiliza ao mesmo tempo mais de uma habilidade classificada como função executiva. (DIAS; SEABRA, 2012).

Para avaliar atenção seletiva descrevem o teste de Atenção por Cancelamento, normatizado no Brasil para crianças a partir de 5 anos até jovens e adultos. (MONTIEL; SEABRA, 2012a). São exigidas atenção seletiva e atenção alternada, envolvendo seletividade ao identificar o estímulo relevante dentre outros, sustentação ao manter a atenção seletiva pelo tempo necessário à resolução da tarefa proposta e alternância ao alternar o foco de atenção conforme requerido na terceira fase desta tarefa. (LEZAK; HOWIESON; LORING, 2004).

A flexibilidade cognitiva pode ser avaliada pelo Teste de Trilhas (Trail Making Test), adaptado em três versões para a realidade nacional: para pré-escolares (TREVISAN; SEABRA, 2012), com dados normativos para crianças e adolescentes entre 6 e 14 anos (MONTIEL; SEABRA, 2012b), e a versão para pessoas entre 18 e 81 anos (RABELO et al., 2010 citados por CARREIRO et al., 2014). O teste é composto por duas etapas, sendo que em uma primeira parte, os itens de uma mesma categoria (números, por exemplo) são apresentados aleatoriamente em uma folha de papel, sendo solicitado que a pessoa ligue estes elementos em ordem crescente seja de tamanho, ordem alfabética ou numérica. Esta etapa envolve velocidade e busca visual. A segunda etapa consiste em solicitar a realização do mesmo procedimento, porém com um nível de complexidade maior, de coordenar duas sequências crescentes concomitantes - por 
exemplo, A, 1, B, 2, C, 3 e assim sucessivamente. Esta segunda etapa avalia atenção, velocidade e flexibilidade, em alternar e coordenar a atenção entre as duas sequências diferentes e concomitantes.

Um dos testes mais utilizados para avaliar controle inibitório é aquele baseado no paradigma do Efeito Stroop (STROOP, 1935, citado por STERNBERG, 2010) em suas diversas versões. O original e mais tradicional é aquele constituído por pares de cores e nome de cores que não coincidem, devendo a pessoa dizer apenas o nome das cores apresentadas acima em cada par, inibindo os nomes de cores não coincidentes escritos logo abaixo, que é a resposta mais automática para uma pessoa que sabe ler. Outras versões são o Stroop Semântico (com figuras e requerer a nomeação de seu oposto, a exemplo, diante da figura de um sol deverá ser nomeada a palavra lua) e o Numérico (números dentro de quadrados, sendo a quantidade de números colocada dentro de cada quadrado inconsistente com o número ordinal representado), que seguem o mesmo princípio das informações inconsistentes em cada item, requerendo inibição da percepção de parte da informação disponível. O Stroop em versão informatizada, além de permitir o registro de acertos, erros e omissões, permite aferir também o tempo de reação, ou seja, tempo necessário para inibir o estímulo concorrente e prestar atenção seletiva ao estímulo adequado e emitir a resposta mais adequada. (CARREIRO et al., 2014).

Componentes da memória de trabalho podem ser avaliados pelo teste de repetição de palavras e pseudopalavras (SEABRA, 2012) e subteste Dígitos, do WISC III (Wechsler Intelligence Scale for Children). (WECHSLER, 2002). Outro instrumento é o teste de Classificação de Cartas de Wisconsin (CUNHA et al., 2005), para crianças, jovens e idosos, sendo um dos mais utilizados internacionalmente para avaliação das funções executivas, que avalia planejamento, memória de trabalho, flexibilidade cognitiva. Para avaliar planejamento, têm sido utilizadas variantes da Torre de Hannói, um paradigma clássico da psicologia cognitiva para avaliar o tempo e a quantidade de operações utilizadas para se partir de um estado inicial até o objetivo final da tarefa. (POZO, 1998). A fluência verbal semântica pode ser avaliada pela solicitação de recuperação oral do maior número de palavras possíveis que começam com a mesma letra, durante um minuto. Para avaliar tomada de decisões pode-se usar o Iowa Gambling Test, que consiste no uso de quatro baralhos, sendo que dois deles envolvem cartas com maiores ganhos, mas também maiores perdas, além de outros dois baralhos cujas cartas concedem menores ganhos, mas também menores perdas, sendo estes os mais vantajosos. (BECHARA, 2003).

Carreiro et al. (2014) descrevem ainda medidas funcionais, que consistem em indagar pais e educadores, ou mesmo crianças e adolescentes, sobre como estes últimos enfrentam situações do dia a dia que requerem a mobilização das 
funções executivas. Chamam a atenção para a carência de estudos e medidas para as chamadas funções executivas quentes, que envolvem a consciência e o controle cognitivo das emoções, bem como de estudos e medidas com valor ecológico, que simulem situações do cotidiano em que funções executivas são requeridas para seu enfrentamento.

O papel preditor das funções executivas no desempenho escolar (SEABRA et al., 2014a) e a correlação entre funções executivas e desempenho em tarefas de avaliação de habilidades de leitura de palavras e compreensão de textos complexos, escrita e matemática tem sido investigado. A relação entre funções executivas e compreensão na leitura de textos será abordada a seguir.

\section{Processos de funções executivas potencialmente envolvidos na compreensão de leitura}

A compreensão leitora envolve múltiplos processos cognitivos, como vocabulário, memória de trabalho, raciocínio lógico, resolução de problemas e funções executivas. (CORSO et al., 2013b; SEIGNEURIC et al., 2000). Corso et al. (2016) testaram vários modelos de equações estruturais sobre diferentes relações entre nível socioeconômico (NSE), inteligência e funções executivas (variáveis independentes) sobre compreensão leitora. No modelo estrutural de melhor ajuste, o efeito do nível socioeconômico sobre a compreensão leitora é totalmente mediado pelas funções executivas, enquanto que a inteligência não tem efeito significativo sobre a compreensão leitora. As funções executivas (incluindo tarefas de span, memória de trabalho, fluência verbal e inibição), portanto, atuaram como mediadoras da relação entre o nível socioeconômico e a compreensão de leitura textual em crianças de $4^{\circ}$ a $6^{\circ}$ ano do ensino fundamental de escolas públicas e privadas. Neste estudo, NSE explicou $31 \%$ da variância em funções executivas, que, por sua vez, foi responsável por explicar $52 \%$ da variância em compreensão de leitura.

Além de estar evidente esta relação no desenvolvimento típico, estudantes com dificuldades específicas de compreensão de leitura quando comparados com leitores proficientes mostram significativamente desempenho mais baixo em funções executivas. (CUTTING et al., 2009; CORSO et al., 2013b). Corso et al. (2013b) compararam o desempenho de maus compreendedores (compreensão leitora deficitária) e bons leitores em uma bateria de avaliação neuropsicolinguística e encontraram diferenças significativas em memória de trabalho visuoespacial, fluência verbal ortográfica e semântica e escrita de pa- 
lavras e pseudopalavras. Habilidades de planejamento e memória de trabalho foram significativamente associadas com a compreensão de leitura. (SESMA et al., 2009). O componente das funções executivas de planejamento parece comprometido em grande parte das crianças com dificuldades específicas de compreensão de leitura. (LOCASCIO et al., 2010).

Ao investigar a relação entre leitura de texto, memória de trabalho, quociente de inteligência (QI) e vocabulário em crianças de $2^{\circ}$ ao $5^{\circ}$ ano do ensino fundamental de escolas públicas, Piccolo e Salles (2013) mostram que o vocabulário foi melhor preditor do desempenho em leitura de texto do que as medidas de memória de trabalho. No entanto, há evidências de que o executivo central da memória de trabalho tem um papel importante na compreensão da leitura, mantendo ativas as representações mentais na memória de trabalho para o entendimento das informações que ainda necessitam ser processadas no texto lido. (KINTSCH; VAN DIJK, 1978). O componente visuoespacial está envolvido na manutenção de uma representação da página e seu layout e facilita, por exemplo, a movimentação dos olhos com precisão a partir do final de uma linha para o início da próxima. (BADDELEY, 2003).

A relação entre funções executivas (incluindo a memória de trabalho) e a compreensão de leitura textual provavelmente varia conforme a idade/escolaridade da criança/adolescente/adulto e proficiência em leitura. Georgiou e Das (2016), ao estudarem uma amostra de adultos, evidenciaram que apenas o componente planejamento das funções executivas teve uma contribuição significativa para o desempenho em compreensão de leitura. Ao comparar adultos com e sem déficits de compreensão leitora os mesmos resultados se confirmam. Os primeiros apresentam déficits nas habilidades de planejamento.

Em crianças do $5^{\circ}$ ano, Nouwens, Groen e Verhoeven (2016) verificaram que o desempenho na compreensão de leitura foi predito pelas habilidades de funções executivas (flexibilidade cognitiva e memória de trabalho - span auditivo de sentenças ou listening span), assim como pelo vocabulário e reconhecimento de palavras. Em uma análise adicional, os autores perceberam que as habilidades de memória (armazenamento), inibição e flexibilidade cognitiva contribuíram para explicar a variância no desempenho da tarefa de memória de trabalho span auditivo de sentenças (listening span), tendo, portanto, uma contribuição indireta para a compreensão leitora.

No estudo de Potocki et al. (2015) três aspectos foram preditivos do desempenho em compreensão de leitura (para questões inferenciais): memória de trabalho, planejamento e inibição. Este mesmo estudo mostrou que crianças com dificuldades específicas de compreensão de leitura, de $3^{\circ} / 5^{\circ}$ e do $7^{\circ} / 9^{\circ}$ anos (entre 8 e 15 anos de idade), apresentam disfunção executiva nos componentes memória de trabalho, inibição, alternância (shifting) e planejamento. 


\section{Considerações finais}

A compreensão de leitura de textos demanda recursos atencionais e de memória, bem como estratégias de organização e realização de raciocínios, entre outros aspectos, funções executivas que ajudam a autogerenciar e alcançar os objetivos da atividade de leitura. Funções executivas podem estar na base dos programas de intervenção junto a crianças com dificuldades de compreensão leitora, bem como em ações preventivas, escolares e públicas, junto a crianças de nível socioeconômico baixo, como forma de prevenir dificuldades em compreensão leitora. (CORSO et al., 2016).

Como planejamento e regulação são elementos centrais do processo de aprendizagem, se comprometidos podem contribuir para dificuldades neste processo. A compreensão dessas relações é indispensável à prática escolar e aos profissionais que atuam nesta área, em uma perspectiva preventiva ou terapêutica (CORSO et al., 2013; SEABRA et al., 2014a), além de promotoras da proficiência em compreender textos, do desenvolvimento do estudante e de seu sucesso escolar.

\section{REFERÊNCIAS}

BADDELEY, A. D. The episodic buffer: A new component of working memory? Trends in Cognitive Sciences, v. 4, n. 1, p. 417-423, 2000.

BADDELEY, A. Working memory: Looking back and looking forward. Nature Reviews Neuroscience, v. 4, n. 10, p. 829-839, 2003.

BECHARA, A. O papel positivo da emoção na cognição. In: ARANTES, V. A. (Org.). Afetividade na escola: Alternativas teóricas e práticas. São Paulo: Summus, 2003.

CANTIN, R. H., GNAEDINGER, E. K.; GALLAWAY, K. C.; HESSON-MCINNIS, M. S.; HUND, A. M. Executive functioning predicts reading, mathematics, and theory of mind during the elementary years. Journal Experimental Child Psychology, n. 146, p. 66-78, 2016.

CARREIRO, L. R. R.; DIAS, N. M.; MALLOY-DINIZ, L. F.; TREVISAN, B. T. T.; MINERVINO, C. A. da S. M.; ROAZZI, A.; SEABRA, A. G. Testes de funções executivas no Brasil. In: Inteligência e funções executivas. São Paulo: Memnon. 2014. p. 73-94. 
CARRETTI, B.; BORELLA, E.; CORNOLDI, C.; DE BENI, R. Role of working memory in explaining poor comprehenders performance: A meta-analysis. Learning and Individual Differences, v. 19, p. 246-251, 2009.

CHANCE, L. Use cloze encounters of the readability kind for secondary school students. J Read, v. 29, p. 690-693, 1985.

CORSO, H. V.; CROMLEY, J. G.; SPERB, T. M.; SALLES, J. F. Modeling the relationship among reading comprehension, intelligence, socioeconomic status and neuropsychological functions-the mediating role of executive functions. Psychology \& Neuroscience, v. 1, p. 32-45, 2016.

CORSO, H. V.; PICCOLO, L. R.; MINA, C. S.; SALLES, J. F. Normas de desempenho em compreensão de leitura textual para crianças de $1^{\circ}$ ano a $6^{\mathrm{a}}$ série. Psico (PUCRS. On-line), v. 46, p. 68-78, 2015.

CORSO, H. V.; SPERB, T. M.; JOU, G. I.; SALLES, J. F. Metacognição e funções executivas: Relações entre os conceitos e implicações para a aprendizagem. Psicologia: Teoria e Pesquisa, v. 29, n. 1, p. 21-29, 2013.

CORSO, H. V.; SPERB, T. M.; SALLES, J. F. Desenvolvimento de instrumento de compreensão leitora a partir de Reconto e Questionário: Estudo preliminar. Neuropsicologia Latinoamericana, v. 4, p. 22-32, 2012.

CORSO, H. V.; SPERB, T. M.; SALLES, J. F. Compreensão Leitora: Modelos de processamento e relações com outras habilidades cognitivas. In: ROAZZI, A.; JUSTI, F. R. dos R.; SALLES, J. F. de. (Org.). A aprendizagem da leitura e da escrita: Contribuições de pesquisas. São Paulo: Vetor Editora, 2013a. p. 83-108.

CORSO, H. V.; SPERB, T. M.; SALLES, J. F. Comparação entre maus compreendedores e bons leitores em tarefas neuropsicológicas. Psicologia em Pesquisa (UFJF), v. 7, p. 37-49, $2013 b$.

CUNHA, J. A.; TRENTINI, C. M.; ARGIMON, I. L.; OLIVEIRA, M. S.; WERLANG, B. G.; PRIEB, R. G. Teste Wisconsin de Classificação de Cartas. São Paulo: Casa do Psicólogo, 2005.

CUTTING, L. E.; MATEREK, A.; COLE, C. A. S.; LEVINE, T. M.; MAHONE, E. M. Effects of fluency, oral language, and executive function on reading comprehension performance. Annals of Dyslexia, v. 59, p. 34-54, 2009. Disponível em: <http://dx.doi. org/10.1007/s11881-009-0022-0>. Acesso em: 14 jun. 2016.

DIAMOND, A. Executive functions. Annual Review of Psychology, v. 64, p. 135-168, 2013.

DIAS, N.; SEABRA, A. Avaliação neuropsicológica cognitiva: linguagem oral. São Paulo: Memnon, 2012. v. 2.

DIAS, N.; SEABRA, A. Programa de Intervenção em Autorregulação e Funções Executivas: Piafex. São Paulo: Memnon, 2013. 
GAUX, C.; GOMBERT, J. E. Implicit and explicit syntactic knowledge and reading in pre-adolescents. British Journal of Developmental Psychology, v. 17, p. 169-188, 1999.

GEORGIOU, G. K.; DAS, J. P. What component of executive functions contributes to normal and impaired reading comprehension in young adults? Research in Developmental Disabilities, v. 49-50, p. 118-128, 2016.

KINTSCH, W.; RAWSON, K. A. Comprehension. In: SNOWLING, M. J.; HULME, C. (Ed.). The science of reading: A handbook. Oxford, UK: Blackwell, 2005. p. 209-226.

KINTSCH, W.; VAN DIJK, T. A. Toward a model of text comprehension and production. Psychological Review, v. 85, n. 5, p. 363-394, 1978.

LEZAK, M.; HOWIESON, M.; LORING, D. Neuropsychological Assessment. 4. ed. New York: Oxford University Press, 2004.

LOCASCIO, G.; MAHONE, E. M.; EASON, S. H.; CUTTING, L. E. Executive dysfunction among children with reading comprehension deficits. Journal of Learning Disabilities, v. 43, p. 441-454, 2010. Disponível em: <http://dx.doi.org/10.1177/0022219409355476>. Acesso em: 02 ago. 2016.

LOGAN, G. Toward an instance theory of automatization. Psychological Review, v. 95, n. 4 , p. $492-527,1988$.

MENEZES, A.; GODOY, S.; TEIXEIRA, L. R. R. C.; CARREIRO, L. R. R.; SEABRA, A. G. Definições teóricas acerca das funções executivas e da atenção. In: SEABRA, A. G.; DIAS, N. M. (Ed.). Avaliação neuropsicológica cognitiva: Atenção e funções executivas. São Paulo: Memnon, 2012. v. 1. p. 34-41.

MIYAKE, A.; FRIEDMAN, N. P.; EMERSON, M. J.; WITZKI, A. H.; HOWERTER, A.; WAGER, T. D. The unity and diversity of executive functions and their contributions to complex "Frontal Lobe" tasks: A latent variable analysis. Cognitive Psychology, v. 41, n. 1, p. 49-100, 2000.

MONTIEL, J. M.; SEABRA, A. Teste de Atenção por Cancelamento. In: SEABRA, A. G.; DIAS, N. M. (Ed.). Avaliação Neuropsicológica Cognitiva: Atenção e funções executivas. São Paulo: Memnon, 2012a. v. 1. p. 57-66.

MONTIEL, J. M.; SEABRA, A. Teste de Trilhas: Parte A e B. In: SEABRA, A. G.; DIAS, N. M. (Ed.). Avaliação Neuropsicológica Cognitiva: Atenção e funções executivas. São Paulo: Memnon, 2012b. v. 1. p. 79-85.

MORAIS, J. A Arte de ler. São Paulo: Editora da Universidade Estadual Paulista, 1996.

NATION, K.; SNOWLING, M. J. Semantic processing and the development of word recognition skills: Evidence from children with reading comprehension difficulties. Journal of Memory and Language, v. 39, p. 85-101, 1998.

NOUWENS, S.; GROEN, M. A.; VERHOEVEN, L. How storage and executive functions contribute to children's reading comprehension. Learning and Individual Differences, v. 47, p. 96-102, 2016. 
PAZETO, T. C. B.; SEABRA, A. G.; DIAS, N. M. Executive Functions, Oral Language and Writing in Preschool Children: Development and Correlations. Paidéia, Ribeirão Preto, v. 24, n. 58, p. 213-222, ago. 2014. Disponível em: <http://dx.doi.org/10.1590/198243272458201409>. Acesso em: 14 jun. 2016.

PICCOLO, L. R.; SALLES, J. F. Vocabulário e memória de trabalho predizem desempenho em leitura de crianças. Psicologia: Teoria e Prática, v. 15, p. 180-191, 2013.

POTOCKI, A.; SANCHEZ, M.; ECALLE, J.; MAGNAN, A. (in press). Linguistic and cognitive profiles of 8 - to 15 -year-old children with specific reading comprehension difficulties: The role of executive functions. Journal of Learning Disabilities, 2015.

POZO, J. I. Teorias Cognitivas da Aprendizagem. 3. ed. Porto Alegre: Artes Médicas, 1998.

SALLES, J. F.; PARENTE, M. A. M. P. Compreensão textual em alunos de segunda e terceira séries: Uma abordagem cognitiva. Estudos de Psicologia (UFRN), v. 9, n. 1, p. 71-80, 2004.

SANTOS A. A. A. Cloze: Um instrumento de diagnóstico e intervenção. São Paulo: Casa do Psicólogo, 2009.

SARAIVA, R. A.; MOOJEN, S. M. P.; MUNARSKI, R. Avaliação da compreensão leitora de textos expositivos para fonoaudiólogos e psicopedagogos. São Paulo: Casa do Psicólogo, 2005.

SEABRA, A. G. Teste de repetição de palavras e pseudopalavras. In: SEABRA, A. G.; DIAS, N. M. (Org.). Avaliação neuropsicológica cognitiva: Linguagem oral. São Paulo: Memnon, 2012. v. 2. p. 97-99.

SEABRA, A. G.; MUNIZ, M.; REPPOLD, C. T.; DIAS, N.; SIQUARA, G.; TOURINHO, A. M. O.; GURGEL, L. G.; TEIXEIRA, L. P. Funções executivas e desempenho escolar. In: Inteligência e funções executivas. São Paulo: Memnon, 2014a. p. 171-182.

SEABRA, A. G.; REPPOLD, C. T.; DIAS, N.; PEDRON, A. C. Modelos de funções executivas. In: Inteligência e funções executivas. São Paulo: Memnon, 2014b. p. 39-50.

SEIGNEURIC, A.; EHRLICH, M. F.; OAKHILL, J.; YUILL, N. Working memory resources and children's reading comprehension. Reading and Writing, v. 13, p. 81-103, 2000 .

SESMA, H. W.; MAHONE, E. M.; LEVINE, T.; EASON, S. H.; CUTTING, L. E. The contribution of executive skills to reading comprehension. Child Neuropsychology, v. 15, p. 232-246, 2009. Disponível em: <http://dx.doi.org/10.1080/09297040802220029>. Acesso em: 02 ago. 2016.

STERNBERG, R. J. Psicologia Cognitiva. São Paulo: Cengage Learning, 2010.

TAYLOR, W. L. "Cloze procedure": A new tool for measuring readability. Journalis Q, v. 30, p. 415-33, 1953. 
TRABASSO, T.; VAN DEN BROEK, P.; SUH, S. Logical necessity and transitivity of causal relations in the representation of stories. Discourse Processes, v. 12, p. 1-25, 1989.

TREVISAN, B. T.; SEABRA, A. G. Teste de trilhas para pré-escolares. In: SEABRA, A. G.; DIAS, N. M. (Org.). Avaliação neuropsicológica cognitiva: Atenção e funções executivas. São Paulo: Memnon, 2012. v. 1. p. 92-100.

VILHENA, D. A.; SUCENA, A.; CASTRO, S. L.; PINHEIRO, Â. M. V. Reading TestSentence Comprehension: An Adapted Version of Lobrot's Lecture 3 Test for Brazilian Portuguese. Dyslexia, v. 22, n. 1, p. 47-63, 2016. Disponível em: <http://www.ncbi.nlm. nih.gov/pubmed/26767907>. Acesso em: 02 ago. 2016.

WECHSLER, D. WISC-III: Escala de Inteligência Wechsler para Crianças: Manual. 3. ed. São Paulo: Casa do Psicólogo, 2002.

Texto recebido em 31 de agosto de 2016. Texto aprovado em 31 de agosto de 2016. 
